West syndrome is a specific, age dependent electroclinical syndrome and one of the most common and well described form of epileptic encephalopathy. In this study, we describe outcome regarding epilepsy and adaptive behavior in patients with WS treated in our clinic.

Patients with WS treated in our clinic from $2000-2018$ were followed up regularly for at least 2 years. For this study, their adaptive behavior was measured using Vineland-II Adaptive Behavior Scales. Adaptive behavior (AB) refers to the skills needed by individuals to function and be self-sufficient within their everyday environments. Vineland-II asses adaptive behavior in four domains: communication, daily living skills, socialization and motor skills, each divided in more subdomains. It provides standardized scores in every domain and is applicable from birth throughout life.

A total of 64 patients were enrolled in this study, 38 males (59\%) and 26 females (41\%). Symptomatic WS had 46 patient $(72 \%)$ and 18 patients $(28 \%)$ had cryptogenic WS. One patient with symptomatic WS died during follow up.

Regardless of response to initial treatment and resolution of infantile spasm, 20 patients (31\%) developed different kind of seizures during follow up and are still taking antiepileptic therapy, 17 of symptomatic (37\%) and 3 of cryptogenic WS (16\%).

Among all patients, $4(6 \%)$ have no deficit in adaptive behavior in all measured domains and subdomains while $40 \%$ of patients have severe or profound deficit. Significant differences were found between symptomatic and cryptogenic form of WS. In cryptogenic WS, most of the patients have moderately low $\mathrm{AB}, 66 \%$ of them, $17 \%$ have $\mathrm{AB}$ and $17 \%$ have low $\mathrm{AB}$. In symptomatic WS moderately low $\mathrm{AB}$ were measured in $37 \%$ of patients, low $\mathrm{AB}$ in $62 \%$ and only $4 \%$ had adequate $\mathrm{AB}$. Low $\mathrm{AB}$ was also in negative correlation with seizure freedom.

Analyzing each domain and subdomain of adaptive behavior, the lowest scores in almost every patient (patients with adequate $\mathrm{AB}$ were excluded) were measured in communication domain, more specific, in expressive speech and in subdomain of community daily living skills.

In this study we confirmed that the most important prognostic factor for outcome of epilepsy and psychomotor development in WS is underlying etiology of the syndrome. Despite the etiology, the great majority (94\%) of the patients with WS need some kind of help in everyday functioning throughout lifetime.

\section{ELECTRICAL STATUS EPILEPTICUS IN SLEEP (ESES): CLINICAL AND EEG CHARACTERISTICS AND RESPONSE TO TREATMENTS}

Sanja Pejic Rosko*, Lana Lončar, Jadranka Sekelj Fureš, Ivana Đaković, Vlasta Đuranović, Katarina Vulin. Children's Hospital Zagreb,Zagreb, Croatia

\subsection{6/archdischild-2021-europaediatrics.399}

Electrical status epilepticus in sleep (ESES) is defined as an age related, self-limited epileptic encephalopathy. It is characterized by heterogeneous clinical manifestations and a specific electroencephalographic (EEG) pattern of continuous spikes and waves during slow sleep (CSWS)

Objective The aim of this study was to describe the electroclinical spectrum in children with electrical status epilepticus in sleep (ESES), and assessment of treatment pattern.
Methods Clinical data of 16 patients with ESES/CSWS syndrome who were treated and followed at least two years were analyzed. Inclusion criteria were as follows: (1) Determination of the ESES pattern on the EEG and a (2) follow-up period of two to four years. All patients underwent a clinical evaluation including history, physical and neurological examinations, sleep and awake EEGs, and brain MRI. Patients with an underlying etiology were classified as symptomatic while others were classified as idiopathic.

Records of EEGs of patients were reevaluated to determine two aspects of ESES: (1) The spike-wave index (SWI) on the NREM sleep EEG and (2) the area of maximum amplitude of continuous epileptic activity. The SWI on the NREM sleep EEG during the ESES period was visually calculated. The ranges of the SWI considered were as follows: N85- 100\% (typical ESES) and 50-85\% (atypical ESES). We also defined the ESES pattern as anterior if the maximum amplitude of spike-waves was in the frontal, frontocentral, or frontotemporal areas and as posterior if the maximum amplitude of spikewaves was in the posterior temporal, temporo-occipital, or occipital areas on the EEG.

Results Complete data were available in 16 children. Age at ESES diagnosis ranged from 36 to 84 months. Antiepileptic drugs were used as first treatment for ESES in 16/16 (100\%). Electrical status epilepticus in sleep initially resolved in $87 \%$, but $56 \%$ had subsequent relapse. At last follow-up, ESES resolved in 56\%. And those children was seizures free.

Conclusion We found high failure rate of first line AEDs in preventing ESES, and high relapse rate. There are no standardizations of managment of ESES.We were managed by our experiences, relevant medical records and clinical trials.

\section{POSTNEONATAL CEREBRAL PALSY - TIMING AND ORIGIN}

Ivana Đaković*, Katarina Vulin, Dolores Petrović, Katarina Bošnjak Nað, Vlasta Đuranović, Jadranka Sekelj Fureš, Sanja Pejić Roško, Lana Lončar, Vlatka Mejaški Bošnjak. Children's Hospital Zagreb, Zagreb, Croatia

\subsection{6/archdischild-2021-europaediatrics.400}

Cerebral palsy (CP) is most common severe neurodevelopmental disorder, affecting 2-3/1000 live borns. Postneonatal cerebral palsy is a rare and distinct form of CP in which damage to immature brain occurs after the newborn period until the age of 24 months. Surveillance of Cerebral Palsy in Europe (SCPE) is European project encompassing 26 currently active centers from 21 European countries. Common database of children with $\mathrm{CP}$ is one of the main assets, providing better insight even in the rare CP types.

Croatian SCPE register is enrolled since 2012 as „C28 RCP-HR Register of cerebral palsy of Croatia', and is affiliated to Children's Hospital Zagreb. Object of this study is to address children with postneonatal origin of CP in our national Register and the main causes of this rare CP.

This population-based study included 502 children from C28 RCP-HR born 2003-2009, living at the area of Register at point of registration, with postneonatal cause of cerebral palsy. Patients were stratified according to the timing and origin of the lesion.

Out of total of 502 children with CP (total CP prevalence 2.11/1000), 11 had postneonatal origin, presenting $2.19 \%$ of total CP population or $0.46 / 10000$ live borns. Most common 
causes were vascular episodes (5/11) and infection of central nervous system (3/11), while others, including the head trauma, accounted for one case respectively. Most usual time of onset was during first trimester (6/11), while timing in other five children was distributed quite equally until the age of two years of life.

Postneonatal cerebral palsy is rare and possibly unrecognised form of cerebral palsy, in our Register, as well as common SCPE register (proportion of $7.69 \%$ of total CP population; prevalence of 1.26/10000). Recognition of this state is important since it is partly preventable, epidemiologically relevant in large scale studies, but most importantly, on individual level, for planning and establishing the special care each CP patient should have.

\section{NEUROSURGICAL TREATMENT OF INTRACTABLE EPILEPSIES DUE TO FOCAL CORTICAL DYSPLASIA}

Sanja Marinković* Vlasta Đuranović. General county hospital Vinkovci

10.1136/archdischild-2021-europaediatrics.401

Focal cortical dysplasia is the most common cause of focal intractable epilepsy in children. According to the ILAE classification there are 3 basic forms of FCD. As diagnostic methods become more sophisticated more often FCD's are recognized as the cause of epilepsy. With the advancement of neurosurgical techniques, surgical procedures are becoming an important element of treating epilepsies.

We would like to represent 2 of our patients with intractable epilepsy due to FCD, who have undergone a neurosurgical evaluation and treatment with excellent results - their epilepsy is cured.

First girl was born from uneventful pregnancy with normal development until the age of 11 months, when seizures began. Her brain CT was normal. As seizures with focal onset repeated, and EEG showed focal changes on the right, anticonvulsive therapy was administrated. Next two years she had just one episode of febrile seizure during illness. Brain MRI done at the age of

4 years was normal. In the next period she had focal seizures with motoric component every couple of months. AET was changed but without clinical improvement. Brian MRI at the age of $9.5 \mathrm{y}$ was normal, but her epilepsy worsened, her focal seizures were often in duration of status epilepticus, so she was referred to Children's hospital Zagreb. Brain MRI done with special sequences shown increased cortical thickness in precentral and postcentral gyri, diagnosis of FCD was made, she was presented to neurosurgical team and after evaluation she was operated. After curative resection, diagnosis of FCD type III B with ganglioma gradus II was made.

After the operation she is seizure free with left sided hemiparesis. Her academic achievements are good.

Second girl was born from uneventful pregnancy and reached her early milestones. At the age of 9 months, she had complex febrile seizure, that repeated at the age of 13 months. Seizures exacerbated during first year of life, so she had many febrile and afebrile seizure episodes, often with duration of epileptic status, without response to antiepileptic therapy.
Neurological decline was evident with left-sided hemiparesis. Brain MRI showed increased cortical thickness on the right precentral gyri and she was referred to neurosurgical evaluation. She was operated and resection of lesion was done, she is now seizure-free, with left-sided hemiparesis.

The lesion showed characteristics of FCD II $b$.

Conclusion Focal epileptogenic lesions can cause generalized epilepsy syndromes in children. The aim of neurosurgical interventions is to eliminate the epileptogenic lesion.

\section{SLEEP DISTURBANCES IN CHILDREN WITH AUTISM SPECTRUM DISORDERS}

Romana Gjergja Juraski*, Matilda Kovac Sizgoric, Mirta Loncar, Ivana Marusic, Marija Milos, Hrvoje Pap, Ines Kunic. Children's Hospital Srebrnjak

\subsection{6/archdischild-2021-europaediatrics.402}

Autism Spectrum Disorders (ASD) are characterized by persistent impairments in reciprocal social interaction and communication. Sleep problems in ASD have significant impact on daily function, social interaction, academic achievements resulting in increased parental sleep disruption and stress.

The genetic factors underlying autism interact in the genesis of the higher percentage of sleep disturbances in children's autism.

During the 4-year-period 64 ASD children were refferred to our Sleep lab and performed an overnight polysomnography (50 males, 14 females, average age

6.76 years, range 2.5-17.5). Inclusion criteria were a diagnosis of ASD using the Diagnostic and Statistical Manual of Mental Disorders IV-R/5 criteria. Exclusion criteria were secondary ASD (e.g. associated with fragile X syndrome, Rett syndrome, Down syndrome etc). Patients with severe physical disability or severe allergy were also excluded from the study.

Polysomnographic parameters included: EMG, EOG, 6-16 channel EEG, nasal pressure, oro-nasal thermistor, chest and abdominal belts for respiratory effort, oximetry with waveform, snoring microphone, EKG, body position, video and audio. Parents filled in the sleep questionnaire.

The analysis revealed that most of the sleep disturbancies included decreased REM quantity, increased undifferentiated sleep, immature organization of eye movements into discrete bursts, decreased time in bed, total sleep time, REM sleep latency, and increased proportion of NREM1 sleep. The sleep fragmentation was higher in children with psychomotoric delay.There was the higher percentage of sleep disruption in the subgroup with focal or/and generalized discharges on EEG, with or without epilepsy.

If treated, the mostly used antiepileptic drugs were valproic acid and levetiracetam.

Polysomnography was useful not only in identifying sleep disorders, but also in demonstrating seizure discharges. Treatment strategies along with limited regulated pharmacotherapy could help improve daily function, social interaction and the quality of life in ASD children and have a beneficial impact on the family. Implementation of bedtime routines and sleepwise approach is still the mainstay of behavioral management in children with autism. 